\title{
Pulmonary Occupancy Combined Brain Abscess Caused by Nocardia Farcinica: Death Case Report and Literature Review
}

\section{Jiangqin Song}

The First People's Hospital of Tianmen City https://orcid.org/0000-0002-6333-4599

\section{Lian Dong}

The First People's Hospital of Tianmen City

\section{Yan Ding}

The First People's Hospital of Tianmen City

\section{Weifang Zhu}

The first people's hospital of tianmen city

Junyang Zhou ( 1466041119@qq.com)

The first people's hospital of tianmen city

\section{Case Report}

Keywords: Nocardia farcinica, brain abscess, death case

Posted Date: July 19th, 2021

DOI: https://doi.org/10.21203/rs.3.rs-675319/v1

License: (c) (i) This work is licensed under a Creative Commons Attribution 4.0 International License. Read Full License 


\section{Abstract}

Background Brain abscess due to the Nocardia genus is rarely reported that usually found in immunocompromised patients. Treatment of Nocardia brain abscess is troublesome and requires consideration of the severity of the underlying systemic disease, the difficulties in identifying the bacterium and the frequent delay in initiating adequate therapy.

Case Presentation Here, we report a rare case of brain abscess caused by Nocardia farcinica. The patient's medical history was complicated, bacterial was found in culture of brain abscess puncture fluid, the colony was identified as Nocardia farcinica by mass spectrometry. Targeted antibiotic treatment was implemented, brain abscess tended to alleviate, but the patient eventually developed fungal pneumonia and died of acute respiratory distress syndrome (ARDS).

Conclusion Early diagnosis, reasonable surgical intervention, and targeted antibiotic treatment are critical for Nocardia brain abscess treatment. Any delay in diagnosis and appropriate therapy can have adverseconsequences.

\section{Background}

Nocardia is an aerobic filamentous environmental grampositivebacterium belonging to the order Actinomycetes. Typically Nocardia is considered as an opportunistic pathogen thatprimarily infects immunosuppressed patients[1]. Nocardial brain abscess is rare and typically found inimmunocompromised patients[1]. Nocardia infections comprise only $2 \%$ of allintracranial abscesses[3], butoverall mortality rate of at least $20 \%[4,5]$. Brain abscesscaused byNocardia farcinica is rarely reported. Treatment of Nocardia brain abscess is troublesome, the difficulties lie in identifying the bacterium, its inherent resistance to certain antibiotics and the frequent delay in initiating the adequate therapy[6, 7].

Here, we report a case of brain abscess caused by Nocardia farcinicain a nonimmunocompromisedpatient, he eventually died of ARDS. Hope to provideexperience for the clinical diagnosis and treatment of Nocardiabrain abscess.

\section{Case Presentation}

A male aged 61 years was admitted to our hospital for intermittent fever and cough on August 16, 2019.In the past 4 years, the patient had developed pulmonary infection repeatedly, which all improved after anti-infection treatment. He had a history of hypertension, coronary heart disease and bronchiectasia. In the past one year, the patient intermittently developed cough, sputum, accompanied by fever, with a body temperature of about $38.0^{\circ} \mathrm{C}$, without afternoon low fever, night sweats and hemoptysis. He was hospitalized for many times, discharged after anti-infection treatment. The patient continued to cough and fever intermittently outside the hospital. 
On admission, the patient was conscious, with no enlargement of superficial lymph nodes, slightly coarse breathing sounds in both lungs, and a little moist crackles could be heard. On June 19, 2019, chest CT (computed tomograph) indicated space occupation in the right upper lung, bilateral lung infective lesion with bronchiectasis, emphysema, bullae of the lung, right pleural effusion Fig. 1A. On August 16, chest CT indicated that the lesion area of the right upper lung mass was significantly larger than before, accompanied by bronchiectasis, emphysema, and pulmonary bulla Fig. 1B. After admission, the patient underwent CT-guided percutaneous lung puncture examination, and the tissues were performed histomathology and microbial culture examination. Histopathology showed chronic inflammatory changes accompanied by mild hyperplasia of alveolar epithelium. No bacterial was observed in lung tissue culture. Bronchoscope alveolar lavage fluid (BALF) examination revealed bronchial inflammation. Cytology of lavage fluid exfoliation: no cancer cells detected;mTB-DNA was not detect in BALF by Gene Xpert. No acid-fast bacilli were found in lavage fluid and sputum by acid-fast staining, and no hyphae and spores of bacteria and fungi were found by gram staining. Mycobacterium culture was negative. $\lg A$, $\operatorname{lgG}, \operatorname{lgM}, \mathrm{C} 3$ and C4 were normal. Blood tests for white blood cells (WBC) $13.6 \times 10^{9} / \mathrm{L}$ (reference

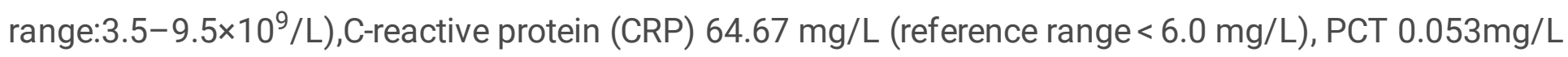
(reference range: $0-0.046 \mathrm{ng} / \mathrm{ml}$ ).

After treatment with cefoperazone sodium + sulbactam sodium + amikacin, the patient's symptoms(fever, cough and sputum) improved, but neurological symptoms such as headache, delirium and memory loss appeared on August 16.A magnetic resonance imaging (MRI) scan of the brain suggested space occupation in the left frontal lobe, the maximum cross-sectional area of the lesion was about $35 \mathrm{~mm} \times 52 \mathrm{~mm}$, brain abscess was considered(Fig. 2.A). On August 28 , the patient underwent minimally invasive puncture drainage under CT-guidance, and about $10 \mathrm{~mL}$ yellow purulent fluid was extracted. The puncture fluid was sent to the microorganism laboratory for testing, after cultured for 48 hours, white cotton-like colonies grew. Aftersmear staining, branching and uneven staining of filamentous bacilli could be seen under the microscope (Fig. 3A-C). The mycelia could be wound into clusters to form actinomycetes like particles, gram stain and the weak acid-fast staining was positive. The bacteria were identified as Nocardia Farcinica by mass spectrometry, $99.9 \%$ credibility. Then, the patient was diagnosed withNocardia farcinica brain abscess. After 16 days of treatment with trimethoprim/sulfamethoxazole $(T M P / S M X)(1.2 \mathrm{~g}-5.0 \mathrm{~g}$, po, bid) and intravenous amikacin $(0.4 \mathrm{~g}$, iv, qd), the patient's temperature returned to normal and his headache completely disappeared, intracranial mass was significantly reduced(Fig. 2.B) and the right upper lung mass was significantly absorbed(Fig. 1.C).During subsequent treatment, the patient developed nausea and vomiting for many times, which was considered to be caused by cerebral edema. After treatment with mannitol dehydration, the symptoms were relieved. Reexamination of head MRI on October 23(Fig. 2.B), it showed that the brain abscess lesions were smaller than before, the brain edema was significantly better than before. Continue with the previous antiinfection treatment regimen.

On December 8, the patient had occasional mild chest tiredness, which relieved spontaneously, intermittent cough, nausea and retching, chest CT showed significant increase in lung lesions, partial 
bronchiectasis, emphysema and bullious lungs appeared. The patient developed dyspnea on December 12accompanied by wheezing sound in both lungs, sputum culture suggested Candida tropicalis, antifungal treatment with itraconazole and doxotheophylline for dyspnea. On December 15,the patient developed ARDS, blood pressure(BP): 100/75mmHg,arterial blood oxygen saturation (SaO2) 79\%.After a series of treatments, including assistant respiration(mask oxygen inhalation), antiinflammatory (methylprednisolone), antiasthmatic (doxofylline, salbutamol, ipratropium bromide), hyperensort(dopamine), the patient's dyspnea symptoms were not relieved and blood pressure did not rise $(\mathrm{BP} 77 / 52 \mathrm{mmHg})$. The patient's family gave up the rescue. Subsequently, the patient goes into a deep coma, loses consciousness, and the heart rate drops. The patient died on the morning of the 16th.

\section{Discussion}

Nocardia is a soil-borne strictly aerobic actynomicete with at least 16 species known to affecthumans[8]. Nocardia spp. have a predilection for the lungs and brain as foci of infection, particularly inimmunocompromised hosts[9].In this case, the patient was a 61-years-old male with no immunodeficiency disease, but he had bronchiectasis, hypertension, coronary heart disease. Multiple lung infections, 11 hospitalizations, and prolonged antibiotic use in the past five years may be key factors in the patient's Nocardia infection

Nocardia farcinica was the most common species, accounted for $24.5 \%$ in Nocardia infection[10]. Nocardia farcinica is more prone to affect the Central Nervous System(CNS )than other species[11, 12]. Clinical manifestations of CNS nocardiosis usually result from local effects of granulomas or abscesses in the brain, which are commonly multiple and, less commonly, the spinal cord or meninges[11, 12]. The abscess usually can be identified by CT scan or MRI as a ring enhancementdepending on the capsular phase [15], but it need to be distinguish with. tumors andcystic or necrotic foci[16]. In our case, after the patient developed symptom of headache, brain abscess was found by MRI examination. Patient underwent minimally invasive surgery for intracranial abscess puncture and suction under CT-guidance, smear staining and bacterial culture were performed on the drainage fluid, and the cultivated colonies were identified as Nocardia farcinica by mass spectrometry.

Nocardia identification can be difficult because of the slowly growing pattern of the germ (colonies usually require at least $48 \mathrm{~h}$ of incubation although more commonly 3 to 5 days and up to 14 to 21 days), preferably in selective media[17]. To Nocardia spp, multiple cerebrospinal fluid (CSF) specimens should be cultured to increase the yield, although it is not uncommon for the bacteria to be recovered only when direct pus is cultured[18]. Certain laboratory techniques like mass spectrometry may help to identify the genus and species. The preferred methods for speciation of Nocardia are 16S rRNA gene analysis and other molecular techniques, such as restriction fragment length polymorphisms and multilocus sequence analysis. Direct abscess drainage seems to be the best method for collection of samples formicrobiological confirmation and antibiotic susceptibility testing[19]. Nocardia pneumonia often requires bronchoscopy or percutaneous lung biopsy, and a detailed history and thorough physical examination should be taken to adequately assess the presence of spread of the lesions. Cranial CT or 
MRI should be performed if symptoms or signs suggest intracranial involvement. The patient was considered to have a pulmonary infection caused by inhalation of the bacterium through the respiratory tract and a cerebral abscess caused by haematogenous spread to the brain. This case was negative in the first percutaneous lung biopsy tissue culture, possibly because no valuable lesion tissue was collected at the biopsy site, leading to the final culture negative. Bacterial grew in the puncture fluid after 24 hours of culture, indicated a severe brain infection and suggested that abscess drainage could also be used to isolate and culture Nocardia.

Direct smears from surgical samples show gram-positive, beaded, branching filaments that are partially acid-fast, and thus need to be differentiated from mycobacteria 1 . Colonies usually have a chalky white cotton-like appearance because of the abundant aerial filaments. The smell of moist or wet soil is very characteristic of Nocardia spp colonies[5]. Nocardia spp exhibit variable morphologic appearances depending on the species, the incubation conditions and the duration of incubation. In routine culture media, Nocardia spp appear as bacillii with ramifications and sub-ramifications at rightangles that may form coccus in Thioglycolate medium after prolonged incubation[8]. The colonies we obtained from the puncture fluid were positive for Gram staining and weak acid resistance for acid-fast staining. The characteristics of bacterial culture and growth (Fig. 3) are consistent with the above literature reports.

Nocardia farcinica brain abscess has a high mortality rate, as high as $20 \%$ in immunocompetent patients and $55 \%$ in immunocompromised patients. These high rates are attributed to the severity of underlying disease, difficulties in identifying the pathogen, and its inherent resistance to antibiotics, leading to inappropriate or late initiation of therapy [6]. In a study of Nocardia isolated from human samples in France, N. farcinica was the most frequently isolated species in blood cultures and brain abscesses $(21 / 39,54 \%$ and $19 / 43,44.2 \%$ respectively. In French data, N. farcinica was frequently not susceptible to cefotaxime ( $80 \%$ of the isolates), meropenem ( $73 \%$ of isolates) and aminoglycosides (more than $90 \%$ ) [20].Taking into account the inherent resistance of Nocardia farcinica to hird-generation cefalosporins, TMP/SMX's ability to cross the blood-brain barrier, most authorities recommend TMP/SMX as part of first-line therapyfor nocardiosis[21, 22]. Abscesses $>25 \mathrm{~mm}$ in diameter and that fail to shrink after $4 \mathrm{wk}$ of antibiotic therapy should be aspirated to confirm the diagnosis regardless of the immune status of the patient [6]. Empiric treatment of cerebral nocardiosis is well established with the use of parenteral TMP/SMX, amikacin, and imipenem-cilastatin[23, 24]. Recently, extended-spectrum fluoroquinolones such as moxifloxacin has been used successfully against N. farcinica cerebral abscess[25]. Because of its ability to cross the blood-brain barrier, TMP/SMX is the treatment of choice and may be effective even when in vitro studies show resistance $[13,23]$. The abscesses in our patient's brain was about $35 \mathrm{~mm} \times 52 \mathrm{~mm}$, far more than $25 \mathrm{~mm}$, minimally invasive puncture drainage is of great significance for the identification of pathogenic bacteria and the treatment of patients. After 16 days of treatment with TMP/SMX, the patient's condition was significantly improved, the lesions in lung and head were also significantly reduced, show a good clinical response (Fig. 1and Fig. 2). This also suggesting that the lung lesions may also be caused by Nocardia infection 
After two months of continuous antibiotic treatment, the patient suddenly developed dyspnea, an acute outbreak of pulmonary fungal infection(Fig. 1), after routine use of antifungal drug, the disease deteriorated and oxygen saturation decreased, eventually resulting in death from ARDS. The patient had recurring lung infections, merge a variety of basic diseases, long-term use of antibiotics, leading to a weakened immune system. When the patient was identified with Nocardia infection, the lung lesions and brain abscesses were severe, which also affected the patient's prognosis.

\section{Conclusion}

Brain abscess caused by Nocardia farcinicain non-immunocompromised individuals is rarely occurs in clinical. In our case, although the patient's condition improved after targeted antibiotic treatment (TMP/SMX), due to lots of basic diseases and long-term use of antibiotics, the central nervous system symptoms appeared lately and delayed diagnosis, the patient eventually died.

For pneumonia of unknown cause, a variety of technical means should be used to determine the pathogen as soon as possible, take targeted treatment, pay attention to the examination of the brain and other organs. Minimally invasive puncture drainage is of great significance for the diagnosis and treatment of Nocardia brain abscess. Because the treatment of Nocardia brain abscess requires longterm use of antibiotics, we should pay attention to the changes of patients' immunity and avoid infection with other pathogens, especially fungi. Early diagnosisand targeted antibiotic treatment are critical for Nocardia brain abscess treatment and prognosis.

\section{Declarations}

\section{Author contributions}

Jiangqin Song and JZ contribute to thesis selection and design, data collection; LD participate in data analysis and interpretation; $\mathrm{RH}$ and $\mathrm{JY}$ contribute to bacterial culture and identification; YD contributes to critical review of the intellectual content of an article; JZ contribute to the manuscript writing.

\section{Funding}

The present study was supported by National Natural Science Foundation of China (81602297), Guangxi Zhuang Autonomous Natural Science Foundation (2018JJB140322)

\section{Data availability}

No additional data are available.

\section{Ethical approval information}

Not applicable. 
Informed consent

The study is supported by the patient's daughter and she hassigned informed consent.

Competing interests

The authors declare that they have no competing interests.

\section{References}

1. Fatahi-Bafghi M: Nocardiosis from 1888 to 2017. Microb Pathog 2018, 114:369-384.

2. Lin YJ, Yang KY, Ho JT, Lee TC, Wang HC, Su FW: Nocardial brain abscess. J CLIN NEUROSCI 2010, 17(2):250-253.

3. Grond SE, Schaller A, Kalinowski A, Tyler KA, Jha P: Nocardia farcinica Brain Abscess in an Immunocompetent Host With Pulmonary Alveolar Proteinosis: A Case Report and Review of the Literature. Cureus 2020, 12(11):e11494.

4. Kumar VA, Augustine D, Panikar D, Nandakumar A, Dinesh KR, Karim S, Philip R: Nocardia farcinica brain abscess: epidemiology, pathophysiology, and literature review. Surg Infect (Larchmt) 2014, 15(5):640-646.

5. Corti ME, Villafañe-Fioti MF: Nocardiosis: a review. INT J INFECT DIS 2003, 7(4):243-250.

6. Mamelak AN, Obana WG, Flaherty JF, Rosenblum ML: Nocardial brain abscess: treatment strategies and factors influencing outcome. NEUROSURGERY 1994, 35(4):622-631.

7. Wilson JW: Nocardiosis: updates and clinical overview. MAYO CLIN PROC 2012, 87(4):403-407.

8. Sabuncuoğlu H, Cibali AZZ, Caydere M, Ustün H, Semih KI: Nocardia farcinica brain abscess: a case report and review of the literature. Neurocirugia (Astur) 2004, 15(6):600-603.

9. Restrepo A, Clark NM: Nocardia infections in solid organ transplantation: Guidelines from the Infectious Diseases Community of Practice of the American Society of Transplantation. CLIN TRANSPLANT 2019, 33(9):e13509.

10. Huang L, Chen X, Xu H, Sun L, Li C, Guo W, Xiang L, Luo G, Cui Y, Lu B: Clinical features, identification, antimicrobial resistance patterns of Nocardia species in China: 2009-2017. Diagn Microbiol Infect Dis 2019, 94(2):165-172.

11. Peters BR, Saubolle MA, Costantino JM: Disseminated and cerebral infection due to Nocardia farcinica: diagnosis by blood culture and cure with antibiotics alone. CLIN INFECT DIS 1996, 23(5):1165-1167.

12. Miralles GD: Disseminated Nocardia farcinica infection in an AIDS patient. Eur J Clin Microbiol Infect Dis 1994, 13(6):497-500.

13. Delvenne E, Farnir F, Guiot J, Giot JB, Von Frenckell C: [Brain abcesses associated with a systemic infection by Nocardia Farcinica]. Rev Med Liege 2017, 72(7-8):340-343. 
14. Buelte D, Noth J, Mull M, Sellhaus B, Koch A, Queider M, Hünger F, Gobbelé R: [Different manifestations of the cerebral nocardiosis]. NERVENARZT 2008, 79(12):1432-1435.

15. Moorthy RK, Rajshekhar V: Management of brain abscess: an overview. NEUROSURG FOCUS 2008, 24(6): E3.

16. Zhu JW, Zhou H, Jia WQ, You J, Xu RX: A clinical case report of brain abscess caused by Nocardia brasiliensis in a non-immunocompromised patient and a relevant literature review. BMC INFECT DIS 2020, 20(1):328.

17. Pascual-Gallego M, Alonso-Lera P, Arribi A, Barcia JA, Marco J: Nocardia farcinica abscess of the cerebellum in an immunocompetent patient: A case report and review of the literature. Asian $J$ Neurosurg 2016, 11(4):454.

18. Chow FC, Marson A, Liu C: Successful medical management of a Nocardia farcinica multiloculated pontine abscess. BMJ Case Rep 2013, 2013.

19. Galacho-Harriero A, Delgado-López PD, Ortega-Lafont MP, Martín-Alonso J, Castilla-Díez JM, Sánchez-Borge B: Nocardia farcinica Brain Abscess: Report of 3 Cases. WORLD NEUROSURG 2017, 106:1015-1053.

20. Lebeaux D, Bergeron E, Berthet J, Djadi-Prat J, Mouniée D, Boiron P, Lortholary O, Rodriguez-Nava V: Antibiotic susceptibility testing and species identification of Nocardia isolates: a retrospective analysis of data from a French expert laboratory, 2010-2015. Clin Microbiol Infect 2019, 25(4):489495.

21. Budzik JM, Hosseini M, Mackinnon AJ, Taxy JB: Disseminated Nocardia farcinica: literature review and fatal outcome in an immunocompetent patient. Surg Infect (Larchmt) 2012, 13(3):163-170.

22. Viganò SM, Edefonti A, Ferraresso M, Ranzi ML, Grossi P, Righini A, Rusconi R, Santambrogio L, Ghio L: Successful medical treatment of multiple brain abscesses due to Nocardia farcinica in a paediatric renal transplant recipient. PEDIATR NEPHROL 2005, 20(8):1186-1188.

23. Brown-Elliott BA, Brown JM, Conville PS, Wallace RJ: Clinical and laboratory features of the Nocardia spp. based on current molecular taxonomy. CLIN MICROBIOL REV2006, 19(2):259-282.

24. Fellows GA, Kalsi PS, Martin AJ: Nocardia farcinica brain abscess in a patient without immunocompromise. Br J Neurosurg 2007, 21(3):301-303.

25. Miksits K, Stoltenburg G, Neumayer HH, Spiegel H, Schaal KP, Cervós-Navarro J, Distler A, Stein H, Hahn H: Disseminated infection of the central nervous system caused by Nocardia farcinica. Nephrol Dial Transplant 1991, 6(3):209-214.

\section{Figures}



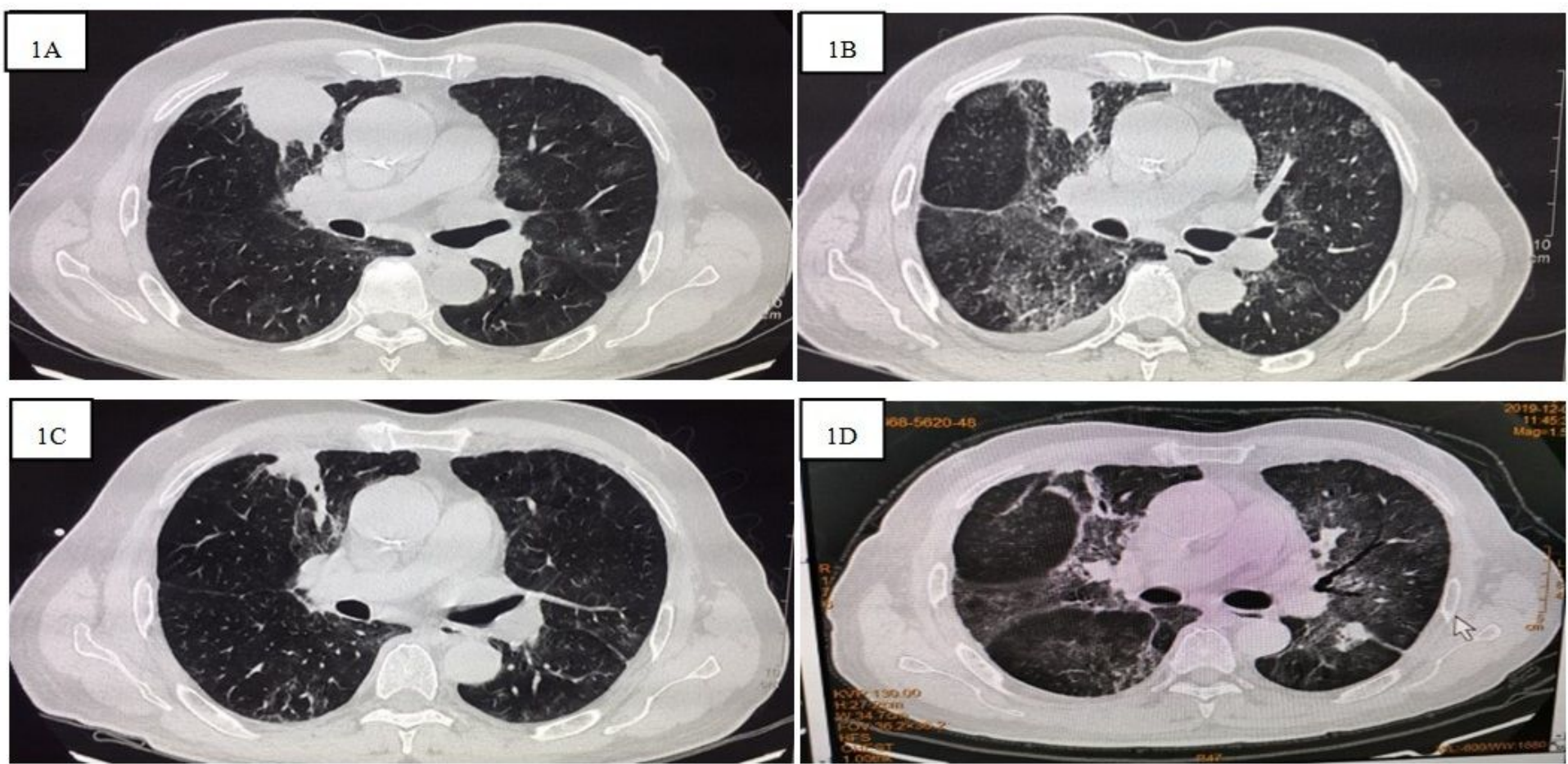

Figure 1

After two months of continuous antibiotic treatment, the patient suddenly developed dyspnea, an acute outbreak of pulmonary fungal infection, after routine use of antifungal drug, the disease deteriorated and oxygen saturation decreased, eventually resulting in death from ARDS
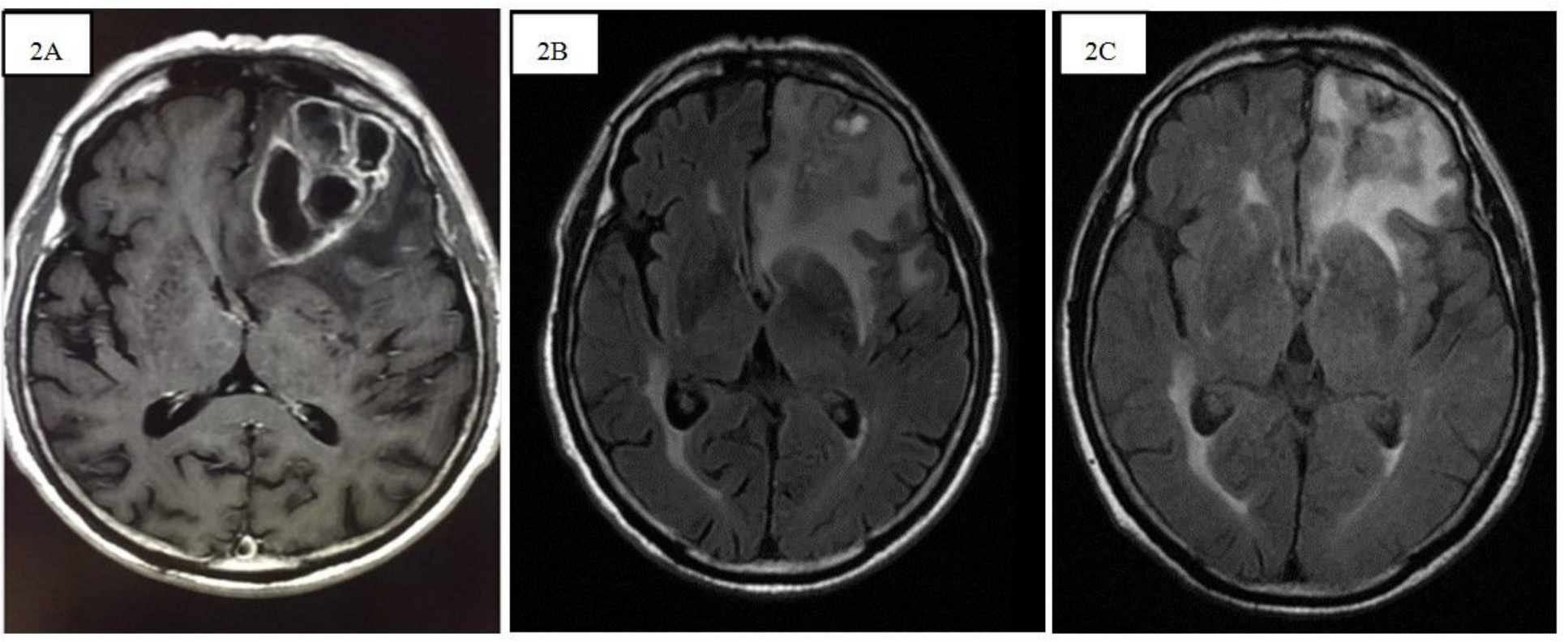

Figure 2

After 16 days of treatment with TMP/SMX, the patient's condition was significantly improved, the lesions in lung and head were also significantly reduced, show a good clinical response. 

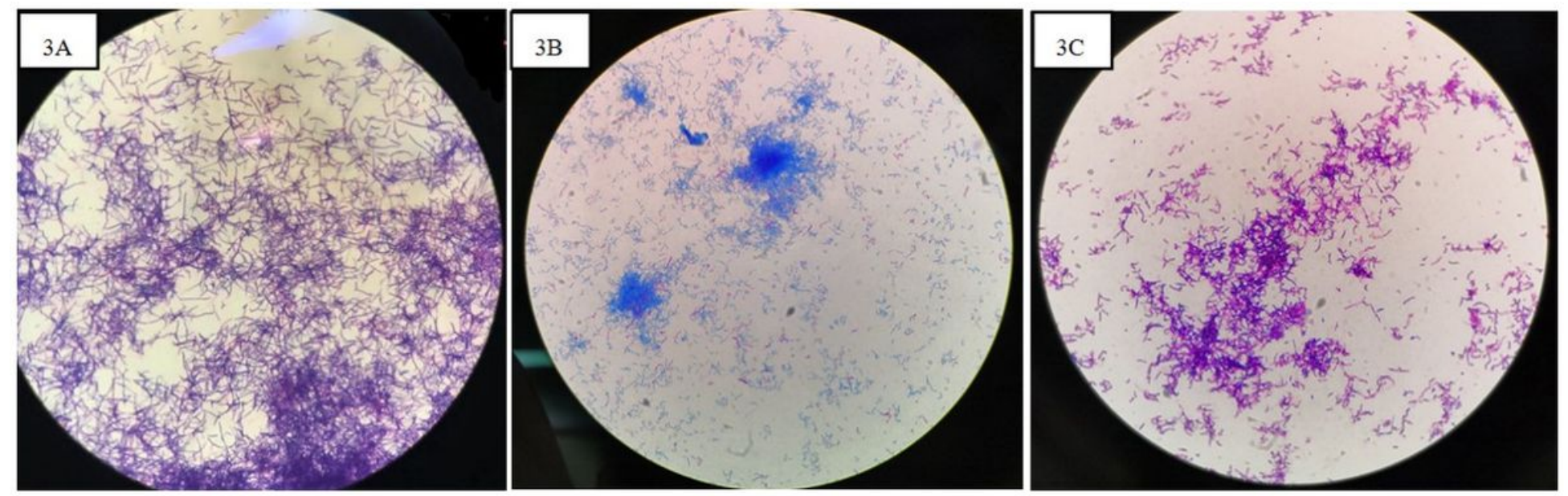

Figure 3

After smear staining, branching and uneven staining of filamentous bacilli could be seen under the microscope (3A-C). 